\title{
Pupillometry: Development of Equipment for Studies of Autonomic Nervous System
}

\author{
Gonçalo Leal $^{1}$, Carlos Neves ${ }^{2}$, and Pedro M. Vieira ${ }^{1}$ \\ ${ }^{1}$ Department of Physics, Faculty of Sciences and Tecnology-UNL, Portugal \\ ${ }^{2}$ Department of Opthalmology, Hospital de Santa Maria, Portugal \\ \{gnl,pmv\}@fct.unl.pt, cmneves@fm.ul.pt
}

\begin{abstract}
This paper presents the results of the development of an equipment for measuring pupil size variation. A Pupillometer has been developed in order to detect the pupil's variation in time in a non-invasive way. The output signal of the equipment expresses the pupil's features variation in time (area, perimeter, vertical diameter, horizontal diameter). The Autonomic Nervous System (ANS) is divided into the Sympathetic and Parasympathetic components and theoretically they are always competing with each other as a two oscillator model. The aim of this project is to identify these components and their frequency bands. With the present results we show that the signal from the pupil's variation has two major frequency bands, the first being the 0-1 $\mathrm{Hz}$ band (LF - Low Frequency) and the second being 1-2Hz band (HF - High Frequency). From the literature we know that the Sympathetic Nervous System (SNS) behaves in a lower frequency than the Parasympathetic (PSNS) one. This means that SNS oscillates in the LF band and PSNS oscillates in the HF band.
\end{abstract}

Keywords: Pupillometry, Edge Detection, Neuro-Physiology, Autonomic Nervous System.

\section{Introduction}

The pupil moves in response to the variation in light intensity in the retina, with a view to assisting the optimizing of visual perception. In dim light, pupil dilation (midriasis) is an effective way to maximize the number of photons reaching the retina, which in turn activates adaptive mechanisms to low light intensity.

Pupil size, shape, and reactivity to light have been used as indicators of neurological function in brain-injured patients, particularly in comatose patients. So, the problem of pupil morphology detection is important for making non-invasive diagnosis of many different diseases, because the pupil of human eyes fluctuates either to adapt the amount of light to the retina or when a subject is gazing at a fixed object in absence of light stimulation and/or visual accommodation [1]. Pupil abnormalities have been widely reported in association with generalized autonomic failure but, except in diabetes, rarely investigated in detail [2]. There are circular and radial muscles that control the size of the pupil. The former is innervated by parasympathetic fibers, and the latter by sympathetic fibers. Thus, the pupil radius is 
controlled by both the sympathetic and parasympathetic nervous systems in response to environmental light, a mechanism called the pupil light reflex. Therefore, the pupillary radius response to an external light stimulus might provide an indirect means to assess the integrity of neuronal pathways controlling pupil size [3].

Pupil's activity is mainly controlled by the Autonomic Nervous System (ANS). The ANS is composed by the Sympathetic Nervous System (SNS) and the Parasympathetic Nervous System (PSNS). We know that the pupil contracts when the Sympathetic component of the ANS is inhibited and the pupil dilates due to the inhibition of the parasympathetic component of the ANS. Because the pupil constrictor muscle is supplied by parasympathetic fibers and the dilator by sympathetic fibers, autonomic disorders may be detected with the appliance of a light stimulus (unresponsiveness to light) or other alarm stimuli.

High-resolution image of the eye necessary for gaze tracking also enables a highprecision measurement of the pupil. This additional measurement is useful, because small, short-term changes in pupil size can indicate cognitive load [4, 5 and 6]. We can see in Figure 1 the changes of pupil's size when a light stimulus was applied to the subject:
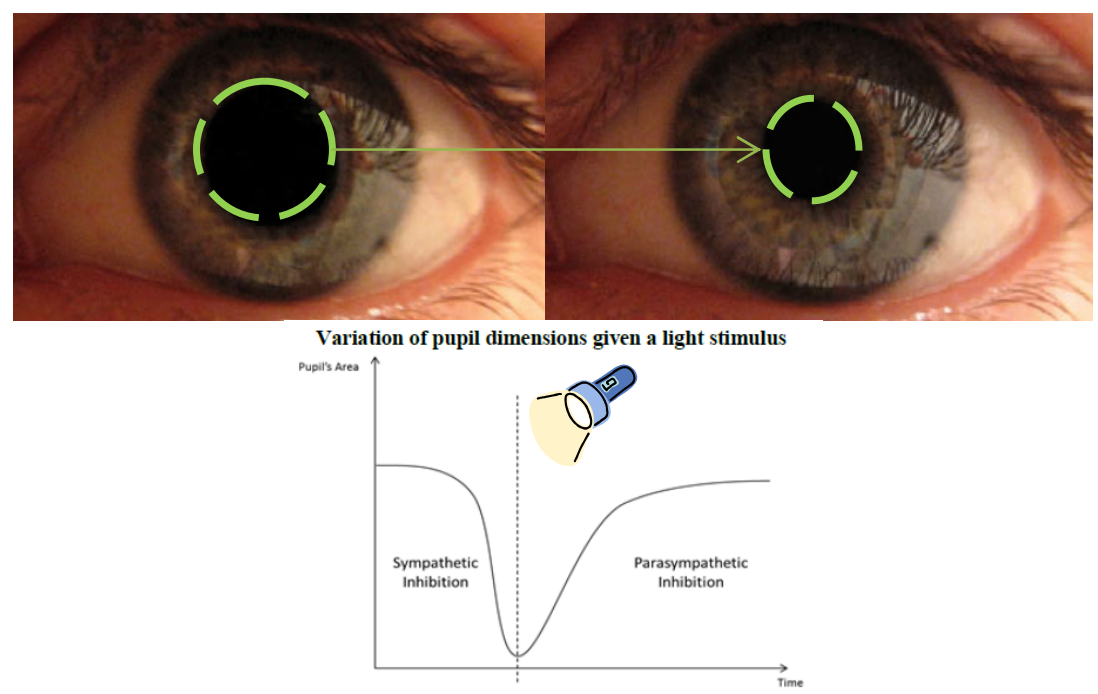

Fig. 1. When the sympathetic system is inhibited we can better see the parasympathetic system activity (HF more evident). When the parasympathetic system is inhibited we can better see the sympathetic system activity (LF more evident). Pictures from http://en.wikipedia.org/wiki/File:Eye_dilate.gif.

If we model this pupil reflex as a system the ANS behaves like a two oscillators model. We have the parasympathetic oscillator and the sympathetic one. These oscillators are competing with each other for predominance. This equilibrium is altered when one of these oscillators is inhibited. This phenomenon allows the other 
oscillator to take over. The inhibition of the sympathetic system is faster compared to the inhibition of the parasympathetic one. We can see this balance disturbed in the pupil. If we apply a light stimulus to the eye, the pupil contracts given to a superimposed central sympathetic inhibition [7]. Then the dilation follows, due both to the parasympathetic relaxation and also the increase of sympathetic activity. By understanding the high frequency structure of these signals we can correlate them to other physiological activity and investigate neurological disorders.

One of the applications of this kind of system is the assessment of Diabetic Autonomic Neuropathy (DAN). DAN is the least recognized and understood complications of diabetes, despite its significant negative impact on survival and quality of life in people with diabetes [8]. It can involve the entire autonomic nervous system. Among the most serious DAN is cardiovascular autonomic neuropathy (CAN), which encompasses damage to the autonomic nerve fibers that innervate the heart, affecting heart rate and blood pressure control and vascular dynamics [3].

This project is the result of a request made by the Department of Physiology of the Institute of Molecular Medicine in Lisbon, to find a way of high rate acquisition and processing of the physiological signals of the eye. The acquisition rate of the equipments that exist on the market is not enough for their research goals, but there are already some studies being made $[1,9,10,11,12,13,14]$.

\subsection{State of the Art}

Several commercial pupillometers exist. They range from specially designed rullers or gauges to highly sophisticated infrared video-based systems. By using gauges we can estimate the pupil diameter with accuracy $\pm 1 \mathrm{~mm}$.

Most accurate systems are based on objective infrared video systems with accuracy of about $0,1 \mathrm{~mm}$. This type of set up is used in toxicology, behavioral science and for drug and alcohol screening.

The growing interest in customized refractive surgeries is not the only factor that drives the need for improved pupillometry. Current advances in soft contact lens correction and human corneal transplantation place a significant demand on eye biometrics in general and particularly on the determination of pupil parameters [14].

\subsection{Physiological Signal Analysis}

The aim of this study is to detect the two main frequency components of the signal of the pupil's size variation given a stimulus. The SNS and PSNS are always present in this signal, but we can force the inhibition of one of them by applying a Sympathetic or Parasympathetic stimulus. Wavelet Analysis is a good method to analyze the signals since they are not stationary.

Many physiological signals may be described either as isolated pulses or as quasiperiodic sequences of isolated pulses. Wavelets are a powerful tool for the representation and analysis of such physiologic waveforms because a wavelet has finite duration (compact support), as contrasted with Fourier methods based on sinusoids of infinite duration [15]. We know that a similar study of the heart and 
lungs has already been made, by decomposing the given signals (ECG and Pneumogram) into a series of sine and cosine functions of different frequencies and amplitudes, allowing the definition of a power spectrum in which three major ranges of frequencies for human subjects can be recognized: very low frequencies (VLF, 0$0.04 \mathrm{~Hz}$ ), low frequencies (LF, 0.04-0.15 Hz) and high frequencies (HF, 0.15-0.4 Hz; Task Force of the European Society of Cardiology and the North American Society of Pacing and Electrophysiology, 1996) [16]. The LF band has been related mainly to sympathetic outflow, while HF is related to parasympathetic outflow and respiratory rhythm. The LF/HF ratio is widely used as an indicator of the balance between sympathetic and parasympathetic outflows [17, 18, 19 and 20].

\section{Methods}

The current system is based on a LE175C LUMENERA camera, which is connected, via Ethernet, to a personal computer. An IR led light ring was used as external illumination and was placed in a mechanical arm attached to a table.

The arm can be moved vertically/horizontally in a mechanical support that moves vertically. An algorithm was developed based on the concept of Intensity Threshold/Region Growing [21].

This experiment was made with a group of subjects with no known physiological pathologies (16 subjects ordered from A to Q). An ECG and a Pneumogram were acquired of each subject during ten minutes in a dark room. After this we applied an eye anesthetic to the subject, in order to give him the ability to withstand his eye open for a long period (60s). We then acquired a pupillogram during 60s. The video files were acquired at the camera's maximum resolution and maximum acquisition rate, $1392 \times 1040$ pixels and $30.3 \mathrm{fps}$, respectively. Afterwards we gave the subject an artificial tear to lubricate the eye and then we acquired a 30 s pupillogram in which we applied 2 light flashes unexpected by the subject to avoid adaptability.

The pupil contour was calculated with an algorithm that preallocates all the frames of an 'avi' file and, given a threshold value, measures the pupil's area, perimeter, horizontal and vertical diameters and the coordinates of the pupil's centroid in each frame.

The algorithm removes the LED ring in a user interface and then uses Threshold fundamentals and edge filters to measure de pupil boundaries. By acquiring the portion of interest it binarizes the image, creates a region and then calculates the mathematical values (see Figure 2). After that it uses the wavelet toolbox functions to plot the original signal along with its frequency components. We then can correlate the acquired signals between each other.

After that, an algorithm was developed in Matlab whose goal is to detect, in a given signal, the amplitude of the frequency bands in time defined by the user [22]. The bands of LF and HF were imputed in the algorithm and, after applying interpolation, the activities of these two bands could be easily analyzed (see table 1). 
Table 1. First approximation of LF and HF bands:

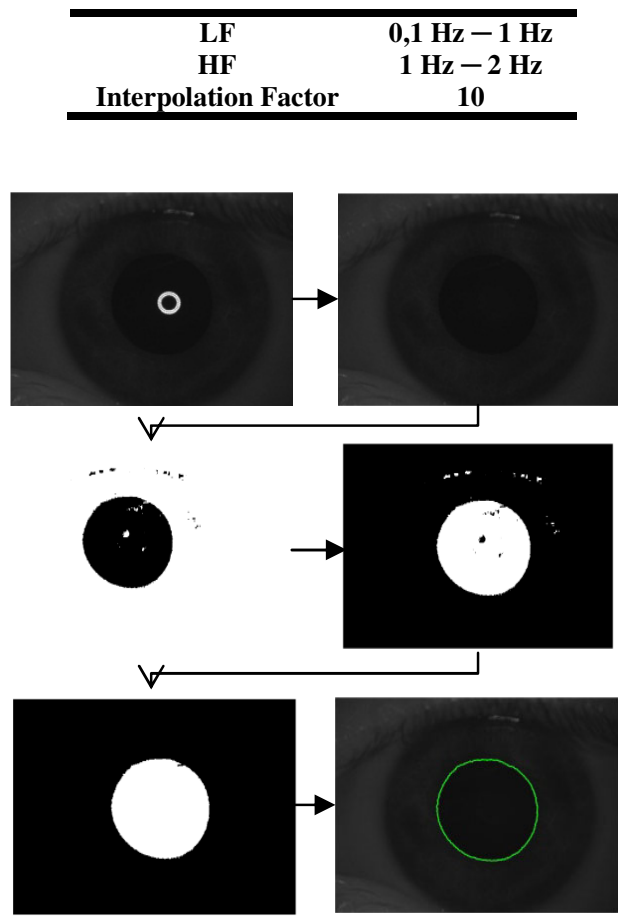

Fig. 2. Detecting the pupil region. First we remove the LED ring illuminator and then we process the image to measure pupil dimensions.

The frequencies of the sympathetic and parasympathetic neural flows in the pupil are unknown because this is a poorly studied topic. So, we made the time-frequency analysis using Wavelets, since we do not have to worry about which frequency windows to choose.

The Wavelet analysis was made using the Matlab Wavelet Toolbox. The Daubechies Wavelet family of order 5 was chosen, since this family is very much used in discrete analysis studies (see Figure 3).

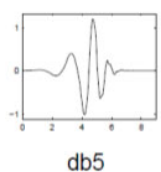

Fig. 3. Daubechies Wavelet family of order 5. [22]

\section{$3 \quad$ Results}

The results will be shown divided into two parts, the first about the pupil detection, using PCD, and the second about the results of the application of Wavelet analysis into the acquired signal from PCD. 


\subsection{Pupil Contour Detection (PCD)}

The pupil contour detection measures the spatial attributes of the pupil and its oscillations. In a video file decomposition is made into each frame that is processed using threshold and region growing methods.

As we can see in Figure 4 the pupil is being detected during time and its data is also saved into a file for post-acquisition processing.
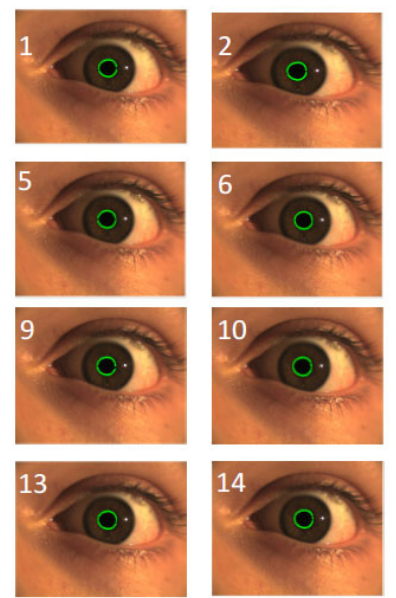
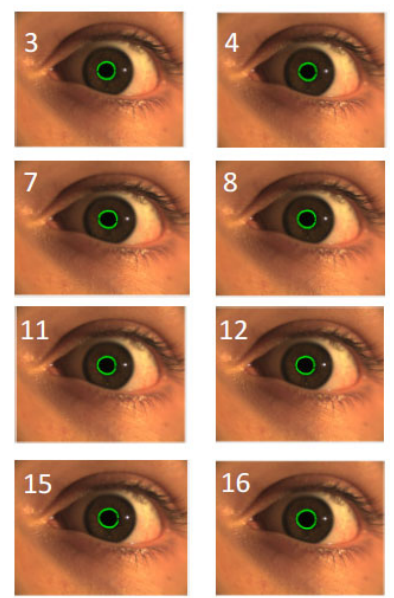

Fig. 4. Example of the PCD output

\subsection{Signal Analysis of the Pupil's Variation in Time}

After the development of the pupil contour detection we applied the developed algorithms to the signals with and without the application of stimuli, as we can see in Figures 5, 6, 7 and 8. As shown in Figure 5, we cannot conclude much of LF and HF activity because the signals have too many oscillations. But, when the stimulus occurs, the subject's pupil contracts and then dilates, giving the signal a high change in amplitude. During contraction the HF band enhances its amplitude and during dilation the LF band is much more evident. As the specialized literature tells us [16, 17], LF has a greater amplitude that of the HF.

After doing the Wavelet analysis, we could see that the Low Frequency signal component (LF) always occurs after the High Frequency one (HF). So the HF peak (in green) could mean the inhibition of the SNS that causes the pupil contraction and LF peak (in red) could mean the inhibition of the PSNS that causes the pupil dilation.

We can also measure the time between the LF and HF peaks of each pupil light reflex.

By analyzing Tables 2 and 3 we can see, after doing peak analysis of the given signals, the difference between the two maximums of each component amplitude enhancement during the stimulus application. Considering some of the results as outliers and also that more subjects must be analyzed, these components are activated with a time interval of about $0.4-0.5$ seconds. 


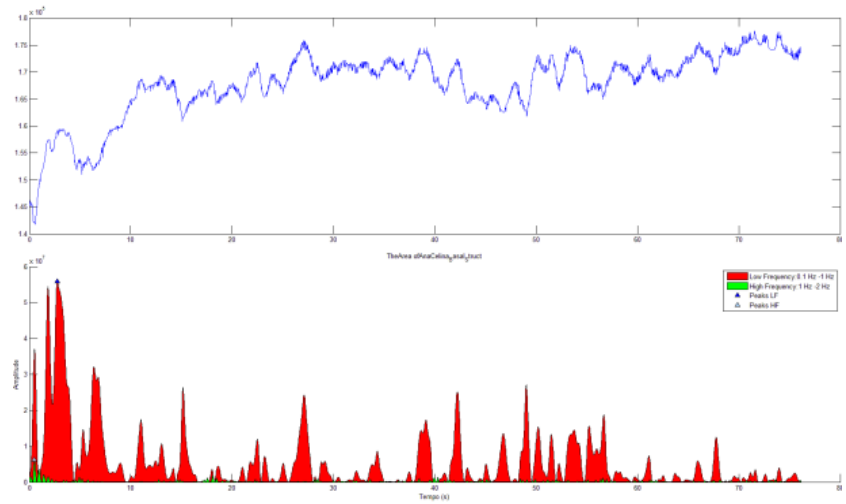

Fig. 5. Pupil's variation of Subject A with no stimuli applied

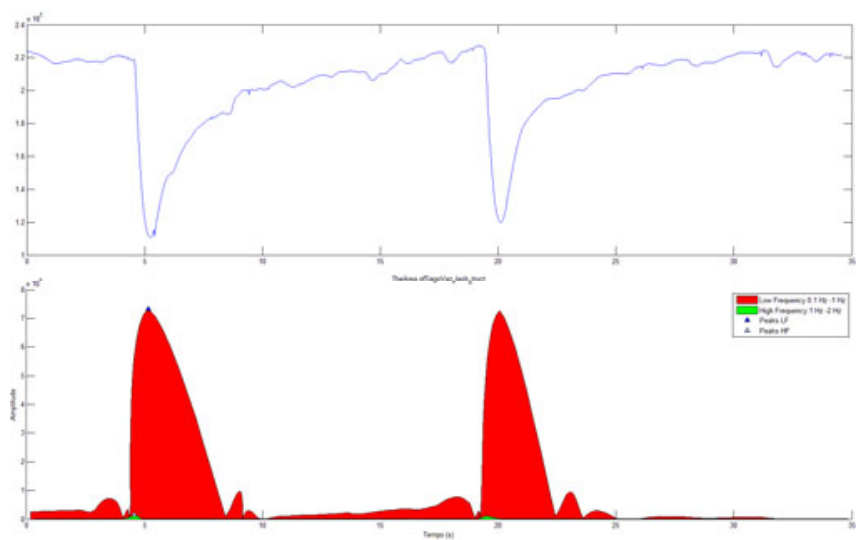

Fig. 6. Application of two stimuli to Subject Q

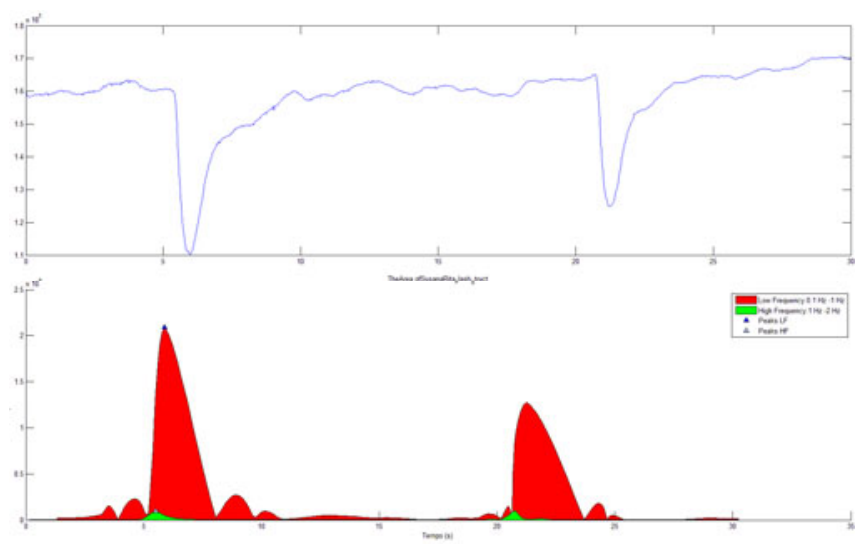

Fig. 7. Application of two stimuli to Subject $P$ 


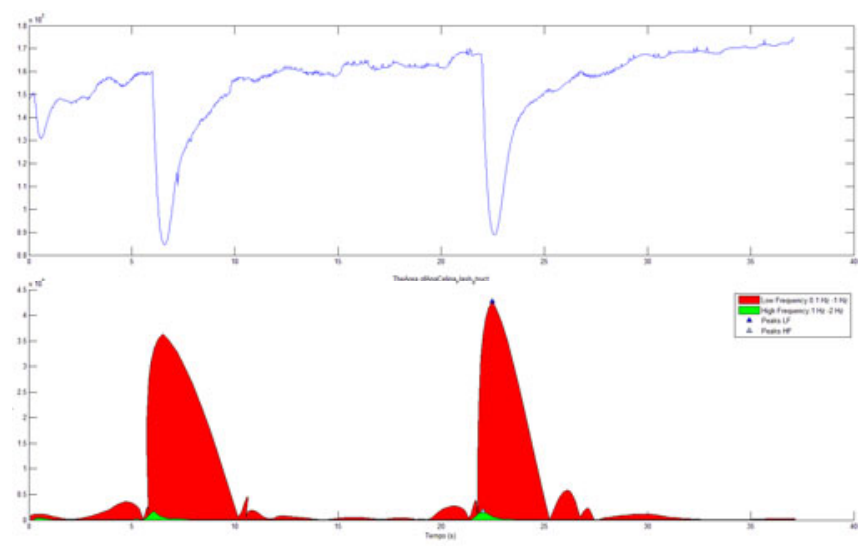

Fig. 8. Application of two stimuli to Subject A

Table 2. Difference between the amplitude maximums occurrence in time:

\begin{tabular}{ccc}
\hline Subject & $\begin{array}{c}\mathbf{1}^{\text {st }} \\
\text { Time Interval } \\
\text { (seconds) }\end{array}$ & $\begin{array}{c}\mathbf{2}^{\text {nd }} \\
\text { Time Interval } \\
\text { (seconds) }\end{array}$ \\
\hline $\mathbf{A}$ & 0,440329 & 0,455194 \\
$\mathbf{B}$ & 0,500000 & 0,500113 \\
$\mathbf{C}$ & 0,766667 & 0,666667 \\
$\mathbf{D}$ & 0,133333 & 0,533333 \\
$\mathbf{E}$ & 0,537448 & 0,370836 \\
$\mathbf{F}$ & 0,600000 & 0,343656 \\
$\mathbf{G}$ & 0,333333 & 0,577874 \\
$\mathbf{H}$ & 0,533333 & 0,338666 \\
$\mathbf{I}$ & 0,466667 & 0,533333 \\
$\mathbf{J}$ & 0,330965 & 0,704767 \\
$\mathbf{K}$ & 0,400000 & 0,313600 \\
$\mathbf{L}$ & 0,0666667 & 0,600000 \\
$\mathbf{M}$ & 0,637085 & 0,600000 \\
$\mathbf{N}$ & 0,100000 & 0,533333 \\
$\mathbf{O}$ & 0,500000 & 0,341336 \\
$\mathbf{P}$ & 0,341391 & 0,365538 \\
$\mathbf{Q}$ & 0,600000 & 0,666667 \\
\hline
\end{tabular}

Table 3. Mean of the peak occurrence:

\begin{tabular}{|ccc}
\hline \multirow{2}{*}{$\begin{array}{c}\text { Mean } \\
\text { (seconds) }\end{array}$} & $\begin{array}{c}\mathbf{1}^{\text {st }} \text { Time Interval } \\
\text { (seconds) }\end{array}$ & $\begin{array}{c}\mathbf{2}^{\text {nd }} \text { Time Interval } \\
\text { (seconds) }\end{array}$ \\
\cline { 2 - 3 } & 0,42866 & 0,49676 \\
\hline
\end{tabular}

\section{Discussion}

A detailed analysis of the results shows that we can relate the pupil's fluctuations to the sympathetic and parasympathetic nervous flows in the subject's brain. This feature can be used to compare the pupil's fluctuations of a normal subject to the ones 
of the pupil of a subject that suffers from a neurological disorder (Alzheimer or Narcolepsy, for example).

The original signal shows us the area of the pupil in pixels ${ }^{2}$ per frame. Since we are studying the pupil's variation, a conversion to millimeters is not needed. By analyzing Figure 5 we see, in the beginning of the pupil contraction, that the detail 3 (d3) has maximum value (white) of scale. This means that this component has higher activity in this area. At the same time the detail 1 (d1) has minimum value (black). When the pupil starts to dilate, $\mathrm{d} 1$ takes a lower value and $\mathrm{d} 3$ takes max value. This behavior occurs in all the processed data, but it is better seen in Flash 1 and Flash 2. These antagonist components can be related to the autonomic activity.

Since the pupil contraction given a light stimuli is a response of an over imposed Sympathetic activity we can better see the different frequency components during the application of a stimuli.

If we apply for example other stimuli, like putting the subject's hand in cold water, we can better see the parasympathetic activity since the pupil dilates.

By comparing our acquisition system to some others made in this area, like IACOVIELLO's in 2006, the innovation is that this system is based not solely in binary transformation (two intensity levels) of the images of the eye but in the transformation of an image to grey levels (255 levels of intensity). This feature allows the detection of small variations in the digital image, so that the error can be reduced and more information gathered.

In a near future we will possibly see two distinct frequency components, one faster than the other, corresponding to the Sympathetic and Parasympathetic Autonomic Nervous Systems, respectively. And we will also be able to correlate the pupil's signal to the ECG from each subject.

It is still not known what kind of frequencies we are looking for. We are now acquiring more data and, by the time of the conference, we intend to present more accurate results and conclusions.

In the future we intend to apply the Pupillometer to subjects suffering from neuroophthalmologic disorders, so that we can infer conclusions and hopefully make the Pupillometer a diagnosis tool. That will make it possible to determine the ratio between the sympathetic and parasympathetic control systems.

Acknowledgments. This project has been funded by the Department of Physiology of the Institute of Molecular Medicine and by the Faculty of Sciences and Technology from the New University of Lisbon. It has been approved by the Santa Maria Hospital (Lisbon, Portugal) Ethics Committee (based on the Laser Products - Conformance with IEC 60825-1 and IEC 60601-2-22; Guidance for Industry and FDA Staff) and is being conducted by a team of 4 physicians and 2 biomedical engineers.

\section{References}

1. Iacoviello, D., Matteo, L.: Parametric characterization of the form of the human pupil from blurred noisy images. Computer Methods and Programs in Biomedicine 77, 39-48 (2005)

2. Bremner, F.D., Smith, S.E.: Pupil Abnormalities in Selected Autonomic Neuropathies. Journal of Neuro-Ophthalmology 26(3) (2006) 
3. Ferrari, G.L., et al.: Using dynamic Pupillometry as a simple screening tool to detect autonomic neuropathy in patients with diabetes: a pilot study. Biomedical Engineering Online 9, 26 (2010)

4. Beatty, J.: Task-Evoked Pupillary Responses, Processing Load, and the Structure of Processing Resources. Psychological Bulletin 91(2), 276-292 (1982)

5. Klingler, J., Kumar, R., Hanrahan, P.: Measuring the Task-Evoked Pupillary Response with a Remote Eye Tracker. In: ETRA 2008 - Eye Tracking \& Research Applications, Savannah, Georgia, March 26-28 (2008)

6. Beatty, J., Lucero-Wagoner, B.: Pupillary System. In: Cacioppo, J.T., Tassinary, L.G., Berntson, G. (eds.) Handbook of Phychophysiology, ch. 6. Cambridge University Press (2000)

7. Heller, P.H., et al.: Autonomic Components of the Human Pupillary Light Reflex. Investigative Ophthalmology \& Visual Science 31(1), 156-162 (1990)

8. Vinik, A.I., Erbas, T.: Recognizing and treating diabetic autonomic neuropathy. Cleve. Clin. J. Med. 68, 928-944 (2001)

9. Smith, S.A., Smith, S.E.: Pupil function: tests and disorders. In: Mathias, C.J., Bannister, S.R. (eds.) Autonomic Failure: A Textbook of Clinical Disorders of the Autonomic Nervous System, 4th edn., Oxford University Press, Oxford (2001)

10. Li, D., Parkhurst, D.J.: Starburst. A robust algorithm for video-based eye tracking

11. openEyes, http://thirtysixthspan. com/openEyes/publications.html

12. Filipe, J.A.C., Reis, F.F., Correia, J.C.: Assessment of autonomic function in high level athletes by pupillometry. Autonomic Neuroscience: Basic and Clinical 104, 66-72 (2003)

13. Hachol, A., et al.: Measurement of pupil reactivity using fast pupilometry. Physiol. Meas. 28, 61-72 (2007)

14. Iskander, D.R., Collins, M.J., Mioscheck, S., Trunk, M.: Automatic Pupillometry From Digital Images. IEEE Transactions on Biomedical Engineering 51(9) (2004)

15. Hohlfeld, R.G., Rajagopalan, C., Neff, G.W.: Wavelet Signal Processing of Physiologic Waveforms. Wavelet Technologies, Inc. (2004)

16. Ducla-Soares, J.L., Santos-Bento, M., Laranjo, S., Andrade, A., Ducla-Soares, E., Boto, J.P., Silva-Carvalho, L., Rocha, I.: Autonomic Neuroscience: Wavelet analysis of autonomic outflow of normal subjects on head-up tilt, cold pressor test, Valsalva manoeuvre and deep breathing. Experimental Physiology 92, 677-686 (2007)

17. Malliani, A.: The sympathovagal balance explored in the frequency domain. In: Malliani, A. (ed.) Principles of Cardiovascular Neural Regulation in Health and Disease, pp. 65108. Kluwer Academic Publishers, The Netherlands (2000)

18. Malliani, A., Pagani, M., Lombardi, F.: Neurovegetative regulation and cardiovascular diseases. Ann. Ital. Med. Int. 6, 460-469 (1991a)

19. Malliani, A., Pagani, M., Lombardi, F.: Physiology and clinical implications of variability of cardiovascular parameters with focus on heart rate and blood pressure. Am. J. Cardiol. 73, 3C-9C (1994)

20. Malliani, A., Pagani, M., Lombardi, F., Cerutti, S.: Cardiovascular neural regulation explored in the frequency domain. Circulation 84, 482-492 (1991b)

21. Leal, G., Vieira, P., Neves, C.: Development of an instrument to measure pupil dynamics in subjects suffering from neuro-ophtalmological disorders. In: Proceedings of the Medical Physics and Biomedical Engineering World Congress 2009, September 7-12, Munich (2009)

22. Misiti, M., Misiti, Y., Oppenheim, G., Poggi, J.-M.: Wavelet Toolbox ${ }^{\mathrm{TM}} 4$ User's Guide. The Mathworks 